\title{
Extensive Epidural Steatitis and Epaxial Myositis Associated with Intramuscular Injection of Melarsomine Dihydrochloride in Two Dogs
}

\author{
Susan A. Arnold ${ }^{1}$ Marc Kent ${ }^{1} \quad$ Jennifer L. Ruby ${ }^{2}$ \\ ${ }^{1}$ Department of Small Animal Medicine and Surgery, College of Veterinary \\ Medicine, University of Georgia, Athens, Georgia, United States \\ 2 Department of Veterinary Biosciences and Diagnostic Imaging, \\ College of Veterinary Medicine, University of Georgia, Athens, \\ Georgia, United States \\ ${ }^{3}$ Department of Pathology, College of Veterinary Medicine, \\ University of Georgia, Athens, Georgia, United States
}

\author{
Lorelei L. Clarke ${ }^{3}$ Renee M. Barber ${ }^{1}$ Simon R. Platt ${ }^{1}$
}

VCOT Open 2020;3:e46-e51.

\begin{abstract}
Address for correspondence Marc Kent, DVM, DACVIM, Department of Small Animal Medicine and Surgery, College of Veterinary Medicine, 2200 College Station Road, Athens, GA 30602, United States (e-mail: mkent1@uga.edu).
\end{abstract}

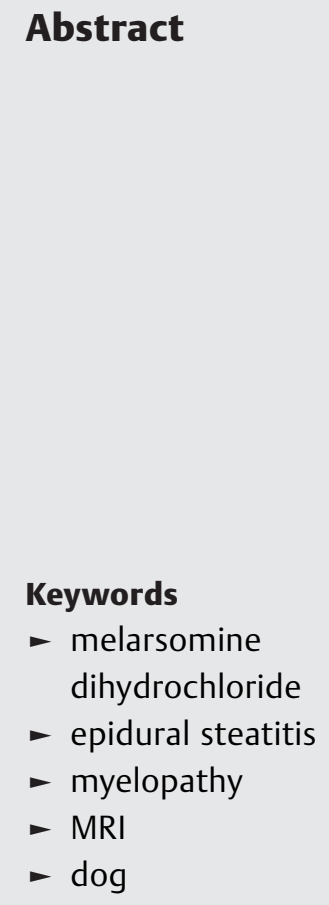

This is a retrospective case series in which clinicopathological and imaging findings associated with neurological complications secondary to melarsomine dihydrochloride administration are reported in two dogs with Dirofilaria immitis infection. Following intramuscular injection of melarsomine dihydrochloride based on manufacturers' guidelines, two dogs developed acute, progressive, nonambulatory paraparesis or paraplegia. Magnetic resonance imaging was suggestive of epidural steatitis, resulting in compression of the spinal cord and spinal cord oedema. In one dog, emphysematous myositis throughout the lumbar paravertebral muscles was present. Hemilaminectomy with the intent of achieving spinal cord decompression and obtaining specimens for microbiological and histopathological evaluation was performed in both dogs. One dog partially regained function postoperatively whereas the other was euthanatized intraoperatively. Histologically, epidural steatitis was observed in the surviving dog and epidural steatitis, myonecrosis and grey and white matter changes in the spinal cord were observed in the euthanatized dog. Microbiological testing failed to identify bacteria from the affected tissues. Both cases highlight the potential for the development of extensive epidural steatitis and consequent spinal cord compression following intramuscular administration of melarsomine dihydrochloride. Further investigation into the optimal therapeutic intervention in dogs experiencing neurological complications following melarsomine administration is warranted.

\section{Introduction}

Melarsomine dihydrochloride, an arsenic-containing anthelminthic used as an adulticide in the treatment of Dirofilaria immitis (canine heartworm), has a narrow safety margin with a complication rate as high as $66 \%{ }^{1-3}$ In addition to common complications of thromboembolism and local irritation at the injection site, melarsomine dihydrochloride administration has been associated with epidural steatitis, resulting in spinal cord compression and paraparesis. ${ }^{2,4,5}$ The use of melarsomine requires strict adherence to administration protocols to minimize risk of complications. Melarsomine is injected deep into the belly of epaxial muscles between the third and fifth lumbar vertebrae. Three injections are performed, alternating sides received

September 25, 2019

accepted after revision

March 21, 2020
DOI https://doi.org/

10.1055/s-0040-1710070. ISSN 2625-2325. (c) 2020 Georg Thieme Verlag KG Stuttgart · New York
License terms

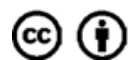


with each injection. The second injection occurs 30 days following the first injection, and the third injection occurs 1 day following the second injection. A 22 gauge, $1 \frac{1 / 2}{2}$ inch needle is used for dogs $>10 \mathrm{~kg}(\mathrm{~kg}){ }^{1}$

This report describes two dogs that developed extensive epidural steatitis and compressive myelopathies. Additionally, one of the dogs developed severe emphysematous myositis of the adjacent lumbar paravertebral musculature. We describe the clinical findings, magnetic resonance imaging (MRI) findings, histopathological findings and outcomes following surgical exploration and decompressive hemilaminectomies.

\section{Case Description}

\section{Case 1}

An adult (unknown age), neutered male $17 \mathrm{~kg}$ Brittany spaniel was evaluated at the University of Georgia (UGA), Veterinary Teaching Hospital for a 2-week history of progressive nonambulatory paraparesis. Two weeks prior, the dog tested positive for Dirofilaria immitis (D. immitis) infection by the referring veterinarian (rDVM) based off $D$. immitis antigen detection in whole blood and received melarsomine dihydrochloride (Immiticide Merial, Duluth, Georgia, United States) in accordance with the manufacturer's instructions ( $2.5 \mathrm{mg} / \mathrm{kg}$ intramuscularly into the left epaxial muscles; this would have been the first of three melarsomine injections. The second injection would have been administered 30 days after the first injection, and the third injection would have been administered 1 day after the second injection). Four minutes later, the dog displayed left pelvic limb lameness. The dog was treated with carprofen $(2.2 \mathrm{mg} / \mathrm{kg}$ per os [PO] every 12 hours) and gabapentin $(10 \mathrm{mg} / \mathrm{kg}$ PO every 8 to 12 hours) for 12 days; however, the signs progressed to nonambulatory paraparesis.

On presentation to UGA physical examination was normal. On neurological examination, the dog was nonambulatory paraparetic. The pelvic limbs had absent postural reactions, reduced patellar and withdrawal reflexes, reduced muscle tone and diffuse muscle atrophy. Anal sphincter tone and perineal reflex were normal. The anatomic diagnosis was consistent with a lesion affecting the spinal cord segments between the fourth lumbar spinal cord segment (L4) through the first sacral spinal cord segment (S1), the respective spinal nerve roots and nerves or the femoral and sciatic nerves. Primary differential diagnoses included spinal cord thromboembolism (vascular differential), epidural steatitis (infectious/ non-infectious inflammatory differential) or direct injection into the vertebral canal (traumatic differential). Other considered differential diagnoses included aberrant heartworm migration, intervertebral disc herniation or meningomyelitis.

A haematology disclosed a leucocytosis (white blood cell count $23.8 \times 10^{3}$ cells $/ \mu \mathrm{L}$; [reference range, 5.5$13.9 \times 10^{3}$ cell $\left.\left./ \mu \mathrm{L}\right]\right) \quad$ with neutrophilia $\left(16.10^{3}\right.$ cells $/ \mu \mathrm{L}$; [reference range, $\left.\left.2.9-12 \times 10^{3} \mathrm{cell} / \mu \mathrm{L}\right]\right)$, elevated band neutrophil count $\left(0.714 \times 10^{3} \mathrm{cell} / \mu \mathrm{L}\right.$; [reference range, 0 $\left.0.45 \times 10^{3} \mathrm{cell} / \mu \mathrm{L}\right]$ ) and a haematocrit of $28.1 \%$ (reference range, 36.6-59.6\%). A serum biochemical profile was normal. A creatine kinase level was not measured.
Under general anaesthesia, MRI (3.0T Seimens Skyra, Siemens Medical Solutions, Malvern, Pennsylvania, United States) of the thoracolumbar vertebral column from the first thoracic (T1) vertebra to the sacrum was performed. The following sequences were acquired: sagittal and transverse T2-weighted (T2W) images using the Dixon technique for fat suppression, two-dimensional multi-echo data image combination images and T1-weighted (T1W) images prior to and after intravenous (IV) administration of gadopentetate dimeglumine $0.2 \mathrm{mmol} / \mathrm{kg}$ (Magnevist, Bayer Health Inc., Whippany, New Jersey, United States). Extending from the tenth thoracic (T10) vertebra to the mid-body of the third lumbar (L3) vertebra, the dorsal epidural space was expanded by abnormal appearing epidural fat that resulted in dorsal extradural compression of the spinal cord. The dorsal epidural fat was heterogeneously T2-hyperintense relative to normal epidural fat and retained a heterogeneous signal intensity on $\mathrm{T} 2 \mathrm{~W}$ images acquired with fat suppression; hypointense areas remained hypointense, whereas some areas of high signal intensity were suppressed compared with T2W images acquired without fat suppression. In other areas, the signal intensity remained T2-hyperintense (-Fig. 1). There was attenuation of the signal from cerebrospinal fluid and epidural fat ventrally. Subjectively, the epidural space lateral to the spinal cord also was expanded bilaterally, giving the appearance of circumferential spinal cord compression. Epidural fat with similar signal characteristics but without compression of the nervous tissues was also present from the L5 vertebra to the lumbosacral junction. From the T4 to T10 vertebrae, the spinal cord was T2-hyperintense without contrast enhancement (-Fig. 1). On two-dimensional multi-echo data image combination images, the epidural fat had hypointense foci consistent with blood products (-Fig. 2). The epidural fat displayed heterogeneous contrast enhancement.

A left-sided hemilaminectomy from T12 to L3 vertebra was performed. The epaxial muscles overlying the L3 and L4 vertebrae appeared dark purple. At the site of spinal cord compression, the epidural fat was tan to dark brown, subjectively with a firm texture and occupying a larger amount of the epidural space than normal, resulting in spinal cord compression. The epidural fat was dissected from the grossly normal appearing dura mater and was submitted for microbiology and histology.

Postoperatively, the dog was treated with fentanyl (2-4 $\mu \mathrm{g} / \mathrm{kg} / \mathrm{h}$ IV as a constant rate infusion) and dexamethasone sodium phosphate $(0.1 \mathrm{mg} / \mathrm{kg}$ IV every 24 hours $)$ for 2 days followed by prednisone $(0.5 \mathrm{mg} / \mathrm{kg}$ PO every 24 hours for 5 days, $0.25 \mathrm{mg} / \mathrm{kg}$ PO for 5 days, $0.25 \mathrm{mg} / \mathrm{kg}$ PO every other day for 5 days).

Aerobic culture of the epidural fat yielded no bacterial growth. Histopathological evaluation disclosed necrotic adipose tissue composed of individual adipocytes surrounded by granular hypereosinophilic necrotic material and variable numbers of degenerate neutrophils.

Three days postoperatively, the dog was less paraparetic but still nonambulatory. Five days postoperatively, the dog was discharged. Six months following initial evaluation, the dog remained nonambulatory paraparetic. 

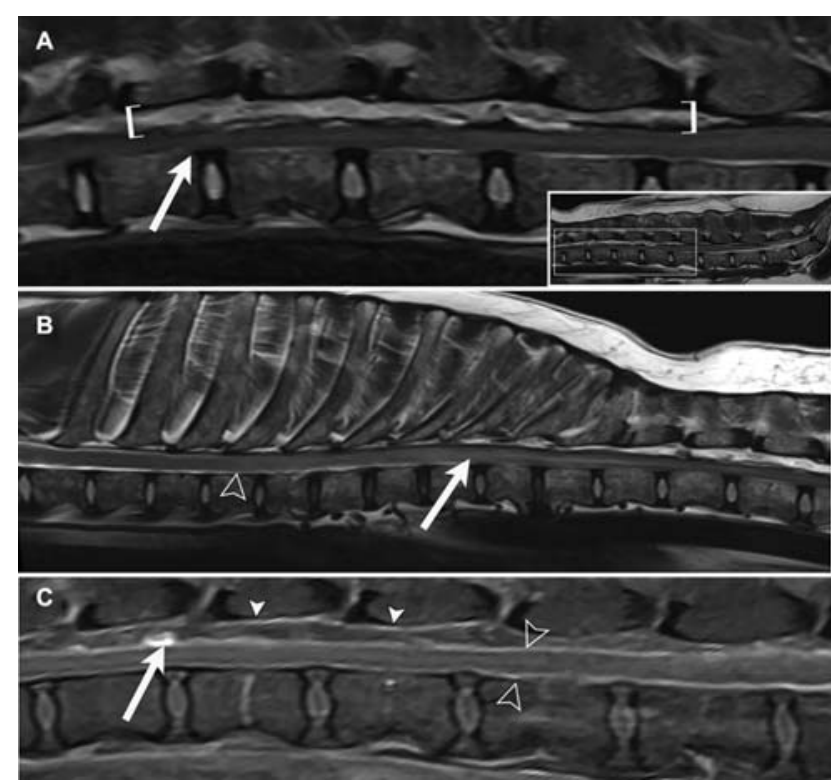

Fig. 1 (A) On the sagittal T2-weighted image from T11 through L3 vertebrae, there is expansion of the dorsal epidural space by epidural fat with a heterogeneous signal intensity (epidural space between the braces) that causes compression of the underlying spinal cord. Areas of spinal cord appear T2-hyperintense (arrow). (B) The T2-hyperintensity in the spinal cord (arrow) extends to approximately the T4 vertebra (open arrowhead) on the sagittal T2-weighted images from $\mathrm{T} 1$ through to the L1 vertebra. (C) Within the region from T11 through L2 vertebra, the post contrast T1-weighted sagittal images obtained with fat saturation reveal enhancement of the meninges (open arrowheads), periosteum of the vertebral canal (white arrowheads) and areas of the epidural fat (arrow).
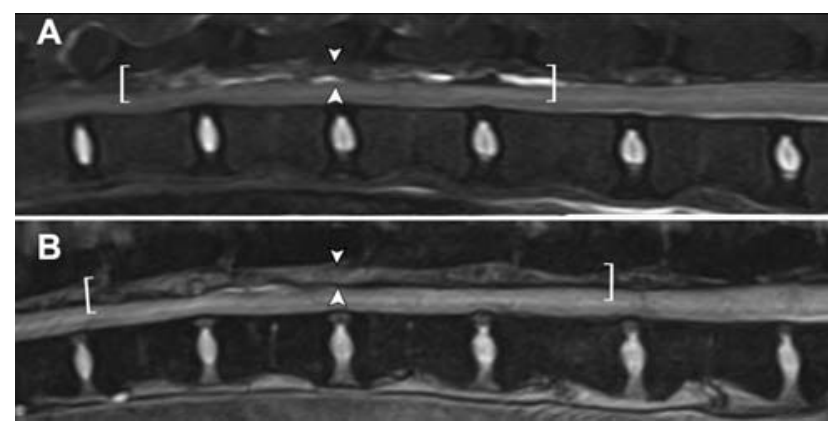

Fig. 2 (A) On the sagittal T2-weighted image with fat suppression from T12 through to the L2 vertebrae, the spinal cord is dorsally compressed by an expanded epidural space (between arrowheads) containing abnormal epidural fat with a heterogenous signal intensity (area between braces) consistent with oedema or cellular infiltrate. (B) Areas of hypointensity within the dorsal epidural fat (affected epidural space is between the braces and arrowheads) on the twodimensional multi-echo data image combination which is consistent with blood products.

\section{Case 2}

An adult (unknown aged) neutered male mixed breed dog weighing $15 \mathrm{~kg}$ was referred for evaluation of a 2-day history of progressive paraparesis. Two months prior, the dog tested positive for $D$. immitis infection and was administered melarsomine dihydrochloride $(2.5 \mathrm{mg} / \mathrm{kg}$ IV in the right lumbar epaxial muscles between the third and fifth lumbar vertebrae) by the rDVM. One month later, a second injection followed
24 hours later by a third injection was administered into the right and left epaxial musculature respectively. Within 18 hours of the third injection, the dog was lame in the left pelvic limb, which progressed to paraplegia over several hours. The following day, the dog was referred.

On physical examination at UGA, the lumbar epaxial musculature was enlarged, painful and had palpable subcutaneous emphysema. The dog's rectal temperature was $40^{\circ} \mathrm{C}$. On neurological examination, the dog was paraplegic with normal pelvic limb sensation. The patellar, withdrawal and perineal reflexes and pelvic limbs muscle tone were reduced. External anal sphincter and tail tone were decreased. The anatomic diagnosis was consistent with a lesion affecting L4 through caudal spinal cord segments, the respective spinal nerve roots, spinal nerves or nerves of the pelvic limbs, anal sphincter and tail. Differential diagnosis was the same as in case 1. However, the presence of tissue gas suggested the presence of anaerobic myositis.

A haematology was normal. Serum biochemical profile disclosed an elevated alanine transaminase (465 U/L; reference range, 10-116 U/L). A creatinine kinase level was not measured. The dog's urine was red coloured, and cytologically, red blood cells were absent, supportive of myoglobinuria. Thoracic and abdominal radiographs disclosed gas in the left lumbar epaxial muscles (-Fig. 3 ).

Under general anaesthesia, MRI of the thoracolumbar vertebral column from the T1 vertebrae to the sacrum was performed. The same sequences were acquired as in case 1 with the addition of a three-dimensional half-Fourier acquisition single-shot turbo spin echo sequence. From the level of the T12 vertebra to the sacrum, the axial muscles were enlarged and had heterogeneous signal intensity on T2W and T1W images. Areas of signal void were present in the subcutaneous tissues and axial muscles on all sequences, consistent with the gas visualized radiographically. Like case 1 , the epidural fat had heterogeneous signal intensity. Subjectively, the epidural space was enlarged, with multifocal areas of T2-hypointense issue, giving the appearance of circumferential spinal cord compression. The spinal cord was hyperintense from the T9 to L4 vertebrae on a three-dimensional half-Fourier acquisition single-shot turbo spin echo sequence (-Fig. 4).

Surgical exploration was pursued with the intent to debride and irrigate presumed anaerobic infection in the epaxial muscles, to decompress the spinal cord and to obtain specimens for microbiological and histopathological evaluation. The subcutaneous fat and epaxial muscles had a haemorrhagic appearance, yet bleeding of incised tissue was not observed. The epaxial muscles were diffusely brown coloured, soft and malodorous. With palpation, gas bubbled up through the muscle. The entire left epaxial muscle system from the $\mathrm{L} 1$ to the L5 vertebrae was resected. A left hemilaminectomy was made from the L1 to the L5 vertebrae. Subjectively, the cancellous vertebral bone did not bleed. The epidural fat was firm, tan and easy to remove (-Fig. 5). Specimens of muscle and epidural fat were submitted for microbiology and histopathology. Given the gross appearance of widespread avascular necrosis of the tissue and the severity of the dog's neurological signs, the prognosis was considered poor. Consequently, the 


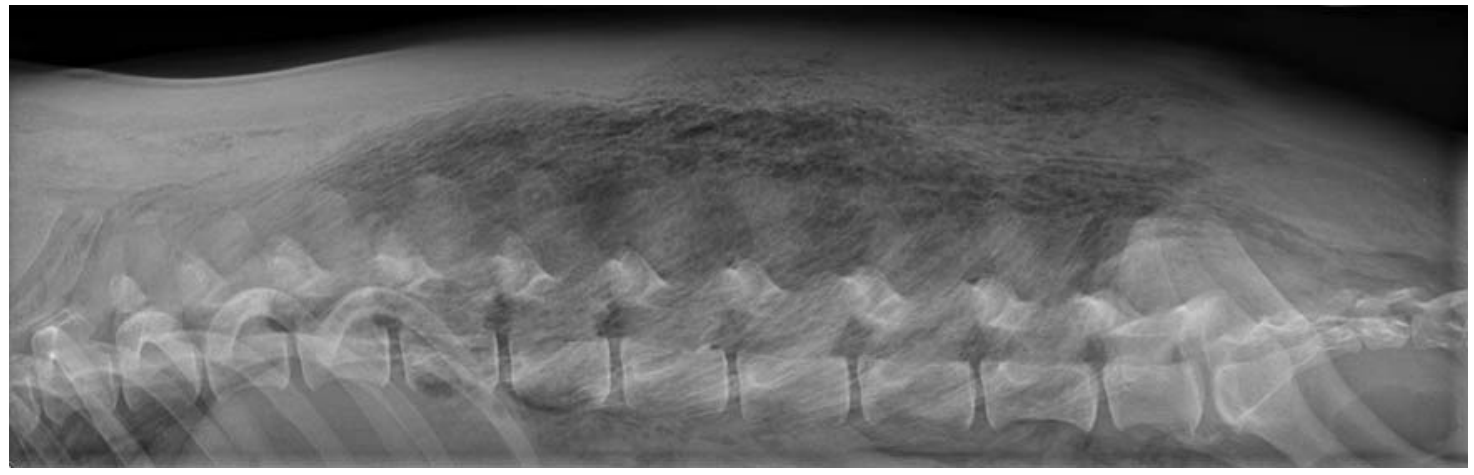

Fig. 3 Lateral radiograph. Gas within the musculature of the lumbar vertebral column is evident on a lateral radiograph from T10 through the sacrum and first two caudal vertebrae.
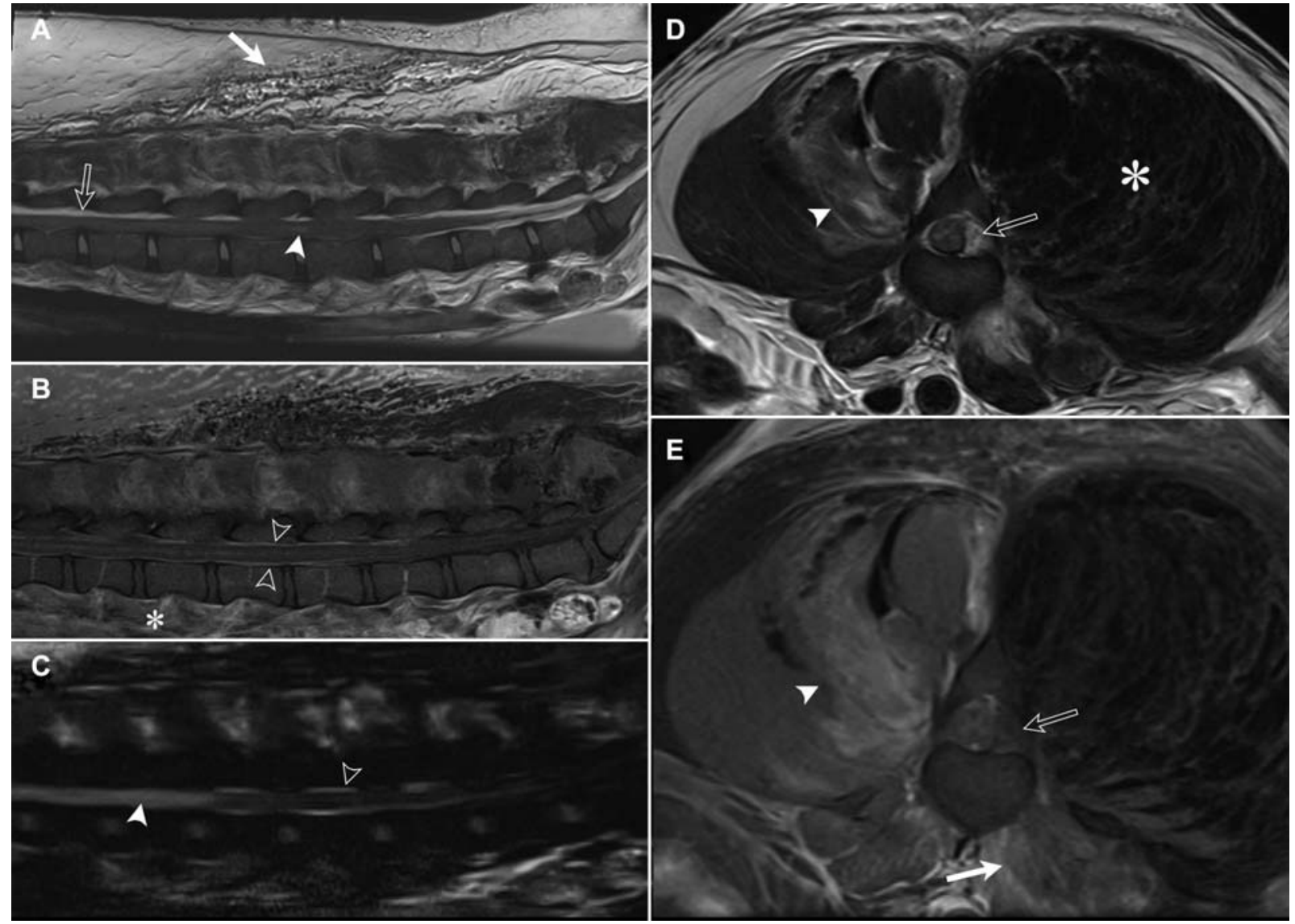

Fig. 4 (A) A sagittal T2-weighted image from the midbody of T11 through the L3 vertebra. The spinal cord is compressed ventrally by the dorsal epidural space which is expanded by abnormal epidural fat (open arrow). Areas of T2-hyperintensity are present in the spinal cord (arrowhead). Punctate hypointensities (signal void) are seen in the subcutaneous fat (white arrow) consistent with gas within the tissue. (B) Enhancement of the meninges (open arrowheads), the spinal cord, areas of epidural fat and the lumbar hypaxial (asterisk) is observed on post contrast T1-weighted images with fat saturation. (C) Hyperintensity of the spinal cord consistent with oedema is more conspicuous using a three-dimensional half-Fourier acquisition single-shot turbo spin echo sequence sequence (white arrowhead). (D) On T2-weighted transverse images at the level of the caudal aspect of the L3 vertebra, the left epaxial muscles are swollen and hypointense (asterisk) compared with the right side. There are focal areas of oedema in the right epaxial muscles (arrowhead). The spinal cord is deviated to the right by heterogeneously hyperintense epidural fat (open arrow). (E) On the T2-weighted transverse image using fat suppression at the same location as in panel D, there is contrast enhancement present in the epaxial (arrowhead) and hypaxial muscles (white arrow) with faint enhancement also present in the epidural space (black arrow).

owner elected humane euthanasia via overdose injection of pentobarbital solution.

At necropsy, the remaining left epaxial and hypaxial muscles from the level of the last rib to the ilium were grossly oedematous, mottled brown, friable and malodorous.
There was a rent in the peritoneum underlying the necrotic muscle, allowing visualization of the abdominal cavity. There was multifocal to coalescing haemorrhage in the left retroperitoneal space. The epidural fat along the lumbar spinal cord was also oedematous and congested. There was no 


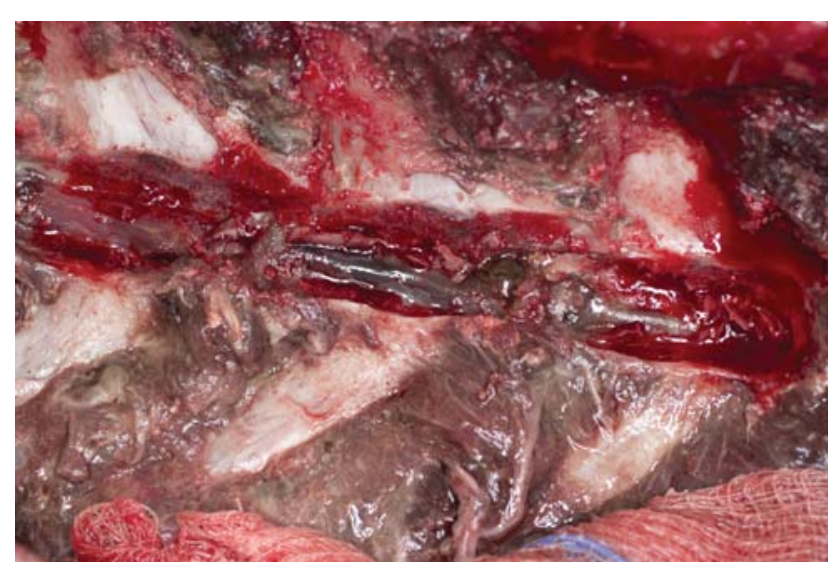

Fig. 5 Intraoperative photograph of the L2 through L4 vertebra of dog in -Figs. $\mathbf{3}$ and $\mathbf{4}$ during a hemilaminectomy. The epidural fat is diffusely discoloured ranging from erythematous at $L 2$ to dark greyblack at L3 and L4. Between the transverse processes, the hypaxial muscles are brown, necrotic and lack active bleeding on cut surfaces. Cranial is to the left of the image.

growth on aerobic and anaerobic cultures on post-mortemcollected affected muscle, and Clostridium chauvoei fluorescent antibody test was negative as well.

On microscopic evaluation, widespread fibrinoid vascular necrosis with multifocal thrombi and haemorrhage was present in the epaxial muscles, obliterating myofiber architecture. In less affected epaxial muscle, necrotic debris, degenerate neutrophils, fibrin and foamy macrophages expanded the perimysial connective tissue. Acute changes of myofiber degeneration, coagulative necrosis and myofiber drop-out also were present with variable regeneration and fibrosis. The epidural fat was effaced by haemorrhage admixed with fibrin, cellular debris, neutrophils, lymphocytes, macrophages and plasma cells. Inflammation infiltrated the dura mater and surrounded the spinal ganglia. Haemorrhage was present in the lumbar spinal cord grey matter along with myelin sheath swelling and spheroids. No bacteria were visualized histologically.

\section{Discussion}

To the authors' knowledge, the severity and extent of the clinicopathological and imaging findings on MRI involving the epaxial muscles, epidural space and the spinal cord have not been reported as a complication to melarsomine injection. Previous reports have documented focal epidural steatitis in proximity to the injection site, thought to be a result of drug extension along spinal nerves, direct injection into the vertebral canal or aberrant heartworm larval migration. ${ }^{4-6}$ In contrast, the dogs herein had extensive epidural steatitis and a consequent compressive myelopathy. Transverse myelitis is a rarely reported complication described in people receiving intramuscular injections of steroids or antibiotic medications with aberrant injection into nerve roots. ${ }^{7}$ Ultimately, the MRI findings combined with the progressive course of clinical signs provided the impetus for pursuing extensive surgical decompression along the entire length of compression of the spinal cord identified on MRI.
Decompressive surgery has resulted in improved neurological function in dogs with neurological dysfunction secondary to melarsomine. ${ }^{4,5}$ Although decompressive surgery may have attributed to the improvement in case 1, the impact of corticosteroid therapy postoperatively likely played a role in the dog's improvement. ${ }^{4}$ Given that the dog's ability to walk was not restored, a potentially irreversible pathological effects on the spinal cord likely had occurred.

It is possible that case 2 represents a more severe form of the sterile epidural steatitis and myositis. ${ }^{2,4,5}$ However, the tissue gas is difficult to reconcile without concurrent anaerobic infection. The odour, gross and histological findings of the epaxial muscles were suggestive of infection with a Clostridium species. Although bacteria were not isolated, this may have been a consequence of aerobic culture of an anaerobic bacterial species.

The source of an anaerobic infection may have been due to contamination of the drug vial, needle and/or syringe, which were unfortunately discarded and not cultured. However, in horses, intramuscular injection-associated clostridial myositis is thought to occur as a result of activation of dormant intramuscular clostridial spores..$^{8-10}$ Muscular injury, such as an injection or tissue reaction to a drug, may lead to necrosis, which creates an anaerobic environment that favours proliferation and exotoxin formation. ${ }^{8}$

The MRI findings aided in determining the extent of the hemilaminectomy needed to decompress the spinal cord. The determination for the need and extent of decompressive surgery as well as the time between the development of neurological signs and surgery in order for a successful outcome necessitates more experience. The lack of improvement in the one dog surviving surgery questions the utility of surgery in dogs with severe clinical signs. The development of fever, myalgia and tissue emphysema at an injection site is highly suggestive of Clostridium infection and should prompt immediate action to attenuate myonecrosis. Obtaining specimens for anaerobic culture should be performed in animals with tissue gas accumulation.

This report highlights a severe complication of melarsomine that reinforces its low margin of safety. Strict adherence to manufacture instructions is critical to avoid administration-related complications. Injection into appendicular muscles results in a significant, protracted lameness. Injection into subcutaneous tissues leads to formation of a sterile abscess. Intravenous injection is fatal. ${ }^{1}$ Drug mishandling or inappropriate administration was not suspected in these cases. Therefore, clinicians should be aware that even with correct administration, severe neurological complications can result from use of melarsomine.

\section{Authors' Contributions}

S.A.A contributed to conception of study, study design and acquisition of data and data analysis and interpretation. M.K. contributed to study design and acquisition of data and data analysis and interpretation. J.L.R. contributed to image analysis and interpretation. L.L.C. contributed to analysis and interpretation of microbiological and gross and microscopic analysis and interpretation of specimens. 
R.M.B. contributed to study design, data analysis and interpretation. S.R.P. contributed to study design, data analysis and interpretation. All authors drafted, revised and approved the submitted manuscript.

Funding

None.

\section{Conflict of Interest}

None declared.

\section{References}

1 Nelson CT, McCall JW, Carithers D. Current canine guidelines forthe prevention, diagnosis, and management of heartworm (Dirofilaria immitis) infection in dogs. Am Heartworm Soc Available at: https://www.heartwormsociety.org/images/pdf/2014AHS-Canine-Guidelines.pdf. Accessed April 8, 2020

2 Maxwell E, Ryan K, Reynolds C, Pariaut R. Outcome of a heartworm treatment protocol in dogs presenting to Louisiana State University from 2008 to 2011: 50 cases. Vet Parasitol 2014; 206(1-2):71-77
3 Hoch H, Strickland K. Canine and feline dirofilariasis: prophylaxis, treatment, and complications of treatment. Compend Contin Educ Vet 2008;30(03):146-151, quiz 151-152

4 Hettlich BF, Ryan K, Bergman RL, et al. Neurologic complications after melarsomine dihydrochloride treatment for Dirofilaria immitis in three dogs. J Am Vet Med Assoc 2003;223(10):1456$-1461,1434$

5 Moore SA, Mariani CL, Van Wettere A, Borst LB. Chronic compressive myelopathy and progressive neurologic signs associated with melarsomine dihydrochloride administration in a dog. J Am Anim Hosp Assoc 2013;49(06):389-393

6 Bonawandt KA, Berg JM, Joseph RJ, Stefanacci JD. Intradural dirofilariasis in a dog with chronic cervical pain. J Am Anim Hosp Assoc 2017;53(01):59-63

7 Deshpande DM, Krishnan C, Kerr DA. Transverse myelitis after lumbar steroid injection in a patient with Behcet's disease. Spinal Cord 2005;43(12):735-737

8 Peek SF, Semrad SD, Perkins GA. Clostridial myonecrosis in horses (37 cases 1985-2000). Equine Vet J 2003;35(01):86-92

9 Songer JG. Chapter 10 - Clostridial Diseases of Animals. In: Rood JI, McClane BA, Songer JG, Titball RW, eds. The Clostridia. San Diego: Academic Press; 1997:153-182

10 Ray D, Cohle SD, Lamb P. Spontaneous clostridial myonecrosis. J Forensic Sci 1992;37(05):1428-1432 